\title{
Temperature dependent dislocation bypass mechanism for coherent precipitates in $\mathrm{Cu}-\mathrm{Co}$ alloys
}

\author{
Jae-Hyeok Shim ${ }^{\text {a,b }}$, Hyon-Jee Lee Voigt ${ }^{c}$ and Brian D. Wirth ${ }^{\text {a,d,* }}$ \\ ${ }^{a}$ Department of Nuclear Engineering, University of Tennessee, Knoxville, Tennessee 37922, USA \\ ${ }^{\mathrm{b}}$ High Temperature Energy Materials Research Center, Korea Institute of Science and \\ Technology, Seoul 02792, Republic of Korea \\ ${ }^{\mathrm{c}}$ Department of Materials Science and Engineering, Massachusetts Institute of Technology, \\ Cambridge, Massachusetts 02139, USA \\ ${ }^{\mathrm{d}}$ Nuclear Science and Engineering Directorate, Oak Ridge National Laboratory, Oak Ridge, \\ Tennessee 37831, USA \\ *Corresponding author \\ Email address: bdwirth@utk.edu (B.D.W.)
}

(C) 2016. This manuscript version is made available under the Elsevier user license http://www.elsevier.com/open-access/userlicense/1.0/ 


\begin{abstract}
Molecular dynamics simulations of dislocation interaction with coherent cobalt precipitates embedded in $\mathrm{Cu}-\mathrm{Co}$ alloys reveal a temperature dependent bypass mechanism. Below $300 \mathrm{~K}$, the trailing partial dislocation clearly bypasses the coherent, face centered cubic (FCC) cobalt precipitate by Orowan looping, caused by a reversible structural transformation as the leading partial locally converts the precipitate to the lower-energy hexagonal close packed (HCP) structure. The FCC versus HCP energy difference of cobalt is temperature dependent, and the dislocation bypass mechanism becomes pure shear above $300 \mathrm{~K}$. Based on a combination of inertial effects due to phonon drag and this observed bypass mechanism, we develop a temperature dependent critical resolved shear stress (CRSS) model, which is in excellent agreement with longstanding measurements of the CRSS temperature dependence of $\mathrm{Cu}-\mathrm{Co}$ alloys, and those obtained from MD simulation. The model explains both the CRSS increase at low temperatures and the existence of a peak value around $200 \mathrm{~K}$.
\end{abstract}

Keywords: Precipitation strengthening; Dislocation glide; Critical resolved shear stress; Molecular dynamics simulation; $\mathrm{Cu}-\mathrm{Co}$ alloy 


\section{Introduction}

$\mathrm{Cu}-\mathrm{Co}$ alloys, which exhibit giant magnetoresistance (GMR) behavior as either multilayer thin films [1] or alloys with embedded cobalt nanoparticles [2], have been studied as a model precipitate-hardened alloy system since the 1950's [3-9]. Ferromagnetic cobalt has limited solubility in copper, and a relatively small lattice mismatch $(<2 \%)$. Thus, cobalt precipitates rapidly form during thermal aging from super-saturated solutions and maintain a coherent, face-centered cubic (FCC) transition phase until losing coherency at larger sizes and transforming to the equilibrium hexagonal close-packed (HCP) structure. Transmission electron microscopy (TEM) studies of the shape, interfacial structure and ripening behavior of Co precipitates in $\mathrm{Cu}$ have shown that the FCC Co particles are roughly spherical and maintain interfacial coherency with a cube-cube orientation relationship until the precipitate size reaches about $10 \mathrm{~nm}[8]$.

Nembach and co-workers $[10,11]$ studied the critical resolved shear stress (CRSS) of $\mathrm{Cu}-\mathrm{Co}$ single crystals and discovered an anomalous temperature dependence. In their studies, coherent cobalt precipitates with a mean diameter of about $6 \mathrm{~nm}$ and estimated to contain $93 \%$ Co were formed by heat treating single crystal $\mathrm{Cu}-\mathrm{Co}$ alloys containing up to 2.2 atomic percent cobalt $[10,11]$. Single crystals were compression tested from 12 to $500 \mathrm{~K}$ at a strain rate of $\sim 1 \times 10^{-4} \mathrm{~s}^{-1}$. Fig. 1 shows the temperature dependence of the CRSS for pure $\mathrm{Cu}$ and alloys containing $0.93,1.28$ and $2.23 \%$ volume fraction of the cobalt precipitates, as reproduced from Fusenig and Nembach [11]. In the precipitate hardened alloys, the CRSS increases with temperature to a maximum value at a temperature around 170 to $230 \mathrm{~K}$, and then decreases at higher temperatures. In these 
alloys, the CRSS at $500 \mathrm{~K}$ is similar to that measured around $10 \mathrm{~K}$. Notably, there is essentially no temperature dependence of the CRSS in the pure $\mathrm{Cu}$ single crystal [11]. Büttner and Nembach [10], and later Fusenig and Nembach [11], attributed the low temperature behavior to dynamic dislocation effects resulting from inertia and phonon drag, which notably disappeared with the addition of increasing concentrations of Au to the $\mathrm{Cu}-\mathrm{Co}$ alloys as a solid solution strengthener [11]. However, the observed CRSS was temperature independent for larger, presumably incoherent precipitates of 40 and $80 \mathrm{~nm}$ diameter over the range of 10 to $300 \mathrm{~K}$ [11]. At higher temperatures, it is generally assumed that thermally activated dislocation climb processes dominate and the material softens [12]. While these mechanisms partially explain the temperature dependence of the CRSS of precipitation hardened $\mathrm{Cu}-\mathrm{Co}$ alloys; the entire temperature dependence and, in particular, the temperature of the maximum CRSS resolved shear stress at a relatively low value of about $200 \mathrm{~K}$ remain to be fully understood.

Complete resolution of the temperature dependence of the CRSS requires detailed atomistic insight into the mechanisms of the dislocation - precipitate interaction. In this paper, we describe large-scale molecular dynamics (MD) simulations of the interaction between gliding dislocations, of edge or screw character, and $3 \mathrm{~nm}$ diameter coherent cobalt precipitates in copper over a temperature range of 10 to $400 \mathrm{~K}$. These results inform a temperature dependent critical resolved shear stress (CRSS) model, based on a combination of inertial effects due to phonon drag and the observed dislocation bypass mechanism, which agrees well with the experimental observations.

\section{Computational methods}


The MD simulations were performed with a modified version of the MDCASK code [13], and used the embedded atom method (EAM) [14] potentials from Foiles et al. [15] and Pasianot and Savino [16] to describe the atomic interactions in pure $\mathrm{Cu}$ and $\mathrm{Co}$, respectively. The $\mathrm{Cu}-\mathrm{Co}$ interactions have been described using the cross pair potential constructed by Nordlund and Averback [17]. They optimized the term so that the potentials can reproduce experimental information such as heat of mixing, lattice parameter and decomposition behavior of $\mathrm{Cu}-\mathrm{Co}$ alloys, which seems to be appropriate for the description of the interface between a Co precipitate and a $\mathrm{Cu}$ matrix. The calculated shear moduli and stacking fault energies of FCC $\mathrm{Cu}$ and FCC Co at $0 \mathrm{~K}$ are presented (Supplementary Table 1). With these potentials, the shear modulus of FCC Co is about two times larger than that of FCC Cu and the lattice parameter of FCC Co is about $2 \%$ larger than FCC Cu. Therefore, modulus mismatch is expected to govern the dislocation - precipitate interaction in the MD simulations, opposite to the experiments where lattice coherency strains are proposed to dominate the dislocation - precipitate interaction [18]. Notably, the stacking fault energy of Co is negative, accurately reproducing the fact that the $\mathrm{HCP}$ Co phase is favored at low temperatures (Supplementary Fig. 1).

The simulation cell consists of an FCC lattice, bounded by ( 111$),(110)$ and $(1 \overline{1} 2)$ faces in $X=a[\overline{1} 11], Y=a / 2[110]$ and $Z=a / 2[1 \overline{1} 2]$ directions, respectively. The cell dimensions for the simulations of edge dislocations are approximately $31 \times 38 \times 27$ $\mathrm{nm}(X, Y$ and $Z$ directions), and contain about 2.7 million atoms; for the simulation of screw dislocations, the cell dimensions are approximately $31 \times 26 \times 66 \mathrm{~nm}(X, Y$ and $Z$ directions), and contain about 4.5 million atoms. Periodic boundary conditions are 
applied in the $Y$ and $Z$ directions. The $X$ surface is initially free, but is subject to a constant surface traction following equilibration. The surface traction provides a shear stress to drive dislocation motion. A coherent FCC Co precipitate about $3 \mathrm{~nm}$ in diameter is inserted symmetrically across the dislocation glide plane, maintaining the cube-cube orientation relationship with the matrix. The precipitate is placed at the center of the cell in the $X$ direction for both edge and screw dislocations. An edge dislocation with $\boldsymbol{b}=a / 2[110](\overline{1} 11)$ is introduced $13 \mathrm{~nm}$ below the precipitate in the $Y$ direction by removing two (220) half planes. A screw dislocation is introduced at the center of the cell based on the continuum elastic displacement field solution for a screw dislocation, which is $16 \mathrm{~nm}$ away from the precipitate in the $Z$ direction. For the edge dislocation, this cell corresponds to a dislocation density of about $8 \times 10^{14} \mathrm{~m}^{-2}$, and a precipitate number density of $3 \times 10^{22} \mathrm{~m}^{-3}$, with a periodic spacing of $27 \mathrm{~nm}$ along the dislocation line. For the screw dislocation, this cell corresponds to a dislocation density of about $5 \times 10^{14} \mathrm{~m}^{-2}$, and a precipitate number density of $2 \times 10^{22} \mathrm{~m}^{-3}$, with a periodic spacing of $26 \mathrm{~nm}$ along the dislocation line. The dislocations naturally dissociate into two Shockley partials with Burgers vectors $\boldsymbol{b}_{\boldsymbol{1}}=a / 6[211](\overline{1} 11)$ and $\boldsymbol{b}_{2}=a / 6[12 \overline{1}](\overline{1} 11)$ with a mean separation of about 7 and $4 \mathrm{~nm}$ for the edge and screw dislocations, respectively, due to the low stacking fault energy of $\mathrm{Cu}$ with this EAM potential [15].

Before applying a shear stress, the cell was equilibrated for $60 \mathrm{ps}$ in the temperature range $10-400 \mathrm{~K}$. No special static relaxation was performed to stabilize the atomic positions around the dislocation. A constant shear stress in the range of $50-100 \mathrm{MPa}$ is applied by superimposing a constant (equal and opposite) force in the [110] direction on the atoms in the outermost (111) surfaces. During the application of the shear stress, the 
velocities of all atoms were re-scaled every 10 steps by multiplying the square root of the ratio of target temperature (average kinetic energy) to current temperature (average kinetic energy) to maintain constant temperature. While the atomic velocity distribution might not precisely follow the ensemble average with this velocity scaling method, the temperature fluctuation due to the application of the shear stress can be minimized. This is an important consideration given that the dislocation bypass mechanism at the precipitate can be very sensitive to temperature change. A time step of 1 fs is used throughout the simulation. The common neighbor analysis (CNA) $[19,20]$ has been used to visualize the dislocation cores. CNA distinguishes close-packed atomic structures such as FCC, HCP and icosahedron from non-close-packed atomic structures by analyzing bond pairs. The dislocation cores have been visualized by selecting atoms with nonclose-packing structures. Visualization of these MD snapshots has been performed using the AtomEye code [21].

We have also developed a phenomenological model to describe the temperature dependence of the CRSS based on our observation of the strongly temperature dependent precipitate obstacle resistance from coherent cobalt precipitates in copper, resulting from this new dislocation bypass mechanism. To account for the inertial effects of phonon drag on dislocation motion, the model incorporates an effective stress contribution to the overall CRSS, based on assuming that the resistance to dislocation motion from the inertial effects of phonon drag can be described by the well-known relation between an effective drag stress and a friction coefficient [22]. Correspondingly, we use a root sum square superposition to define the overall temperature dependent CRSS by combining the 
effective phonon drag resistance stress with the dispersed obstacle strengthening, which captures the temperature dependence of these two different mechanisms.

\section{Results and discussion}

Fig. 2 shows snapshots from the MD simulation of edge dislocation interaction with a $3 \mathrm{~nm}$ Co precipitate at $300 \mathrm{~K}$ and an applied shear stress of $100 \mathrm{MPa}$. During equilibration, the $3 \mathrm{~nm}, \mathrm{FCC}$ Co precipitate maintains coherency with the $\mathrm{Cu}$ matrix, without any indication of impending structural transformation or interface instability. The edge dislocation is noticeably split into two Shockley partials with a mean separation of about $7 \mathrm{~nm}$, which glide in the $Y$ direction. After about $16 \mathrm{ps}$ of applied stress, the leading partial initially contacts the Co precipitate (Fig. 2a). By about 23 ps, the leading partial shears the precipitate, noticeably bowing with a critical angle of about $70^{\circ}$ (Fig. 2b). As the leading partial moves past the particle, the trailing partial begins to interact with the precipitate (Fig. 2c). By about 44 ps, the leading partial has moved through the periodic boundary conditions and re-entered the cell from the bottom (Figs. $2 \mathrm{c}$ and d), while the trailing partial continues to be pinned by the precipitate. About $45 \mathrm{ps}$ after the applied stress, the trailing partial dislocation bypasses the precipitate by the expected shear mechanism (Fig. 2e). Noticeably, the resistance due to the precipitate is stronger for the trailing rather than the leading partial, with a critical bypass angle of approximately $50^{\circ}$ as the trailing partial detaches from the precipitate (Fig. 2e). Considering the atomic arrangement of the Co precipitate before and after during dislocation bypass, the two central atomic layers of the precipitate take an HCP stacking after the bypass of the leading partial, which indicates the precipitate lies on the stacking fault (Supplementary 
Fig. 2). After the bypass of the trailing pass, these atomic layers return to an original FCC stacking out of the stacking fault, but with a sheared shape of the precipitate. A similar process occurs for the $2^{\text {nd }}$ subsequent bypass of the leading and trailing partials. MD simulations at $400 \mathrm{~K}$ reveal a similar sequence of events and interaction/detachment mechanism, namely that both the leading and trailing Shockley partial dislocations bypass the coherent Co precipitate by the expected shear mechanism, with the trailing partial experiencing more resistance. For detailed MD simulation results, see Supplementary Movie 1.

Fig. 3 shows snapshots from the MD simulation at $100 \mathrm{~K}$. The results are similar to those at $300 \mathrm{~K}$ for the leading partial dislocation, but the bypass mechanism for the trailing partial is completely new and unexpected. After about 15 ps of applied stress, the leading partial initially contacts the Co precipitate (Fig. 3a). By about 22 ps, the leading partial shears the precipitate, noticeably bowing with a critical angle comparable to the higher temperature simulation (Fig. 3b). As the leading partial moves past the particle, the trailing partial begins to interact with the precipitate. By about $41 \mathrm{ps}$, the leading partial has moved through the periodic boundary conditions and re-entered the cell from the bottom (Figs. 3c and d), while the trailing partial continues to be pinned by the precipitate, bowing to a critical angle of nearly $0^{\circ}$. As the trailing partial nearly completely surrounds the precipitate (Figs. 3d and e), the partial dislocation dipole segments just beyond the precipitate strongly interact and annihilate. This releases the trailing partial dislocation from the precipitate and leaves an Orowan loop around the precipitate, with partial dislocation character (Fig. 3e). The resulting Orowan loop, with a Burgers vector of $\boldsymbol{b}_{2}=a / 6[12 \overline{1}](\overline{111})$, does not have a perfectly symmetric circular shape, 
but rather is elliptical in which some segments penetrate the precipitate. This glide loop is not energetically stable at this size and collapses, resulting in the additional shear of the precipitate, as the trailing partial completely moves past the particle (Fig. 3f). Viewing the final precipitate configuration from an edge-on, $<111>$ projection (although not shown here), reveals precipitate shear by a full Burgers vector, $\boldsymbol{b}=a / 2[110](\overline{1} 11)$, even though the trailing precipitate did not bypass by the anticipated shear mechanism. For details, see Supplementary Movie 2. The same bypass mechanism also occurs, and is somewhat easier to visualize, for a larger precipitate (Supplementary Movie 3).

Each MD simulation of edge dislocation - Co precipitate interaction with an $100 \mathrm{MPa}$ applied shear stress and temperatures between 10 and $200 \mathrm{~K}$ showed this bypass mechanism involving a mix of shear for the leading partial dislocation and Orowan looping of the trailing dislocation. The different dislocation bypass mechanism for the leading and trailing Shockley partial dislocations, manifested by a different critical bowing angle for each partial, clearly indicates that the Co precipitate offered more resistance to the trailing partial. Even though the resulting Shockley partial (Orowan) dislocation loop collapsed, producing the net result of a sheared precipitate by one perfect Burgers vector, its obstacle strength is clearly temperature dependent, with the precipitate being a stronger obstacle at low temperatures than a simple shearable particle.

An important question to ask is whether this behavior is unique to the edge dislocation? Fig. 4 presents results from the interaction between a screw dislocation and the $3 \mathrm{~nm}$ coherent cobalt precipitate, at a temperature of $100 \mathrm{~K}$ and an applied shear stress of $100 \mathrm{MPa}$. The screw dislocation also dissociates into two Shockley partials, but with a smaller mean separation of about $4 \mathrm{~nm}$. By about $26 \mathrm{ps}$, while the leading partial shears 

contacts the precipitate (Fig. 4a). About 20 ps after the leading partial completely passed through the precipitate by shear, the trailing partial surrounds the precipitate until a critical bowing angle reaches almost $0^{\circ}$ (Fig. $4 \mathrm{~b}$ ). Then, the trailing partial is detached from the precipitate, leaving an Orowan loop around the precipitate (Fig. 4c). Shortly after the trailing partial detaches via the Orowan mechanism, the resulting Orowan glide loop collapses into the precipitate, again resulting in the shear of the precipitate by a full Burgers vector (Fig. 4d). These snapshots indicate that the mixed shear and Orowan bypass mechanism is also true for screw dislocations. For details, see Supplementary Movies 4 and 5.

This new, mixed shear and Orowan dislocation bypass mechanism and the transition in mechanism with temperature observed in MD simulations can be understood by considering the precipitate resistance to dislocation motion. For shearable coherent precipitates, the resistance to dislocation motion results primarily from elastic modulus mismatch or difference in atomic size (or lattice parameter) between the precipitate and matrix, in addition to secondary contributions from interface generation $[18,22]$. None of these mechanisms should differ substantially for the two Shockley partial dislocations, nor have strong temperature dependence. For the specific case of coherent cobalt precipitates embedded in an $\mathrm{FCC} \mathrm{Cu}$ matrix, a new strengthening mechanism must be considered, due to the reversible crystallographic structural transformation of the precipitate, which is different for each of the two Shockley partial dislocations.

As the leading partial shears the precipitate by $a / 6[12 \overline{1}]$, a plane of HCP stacking (e.g., the FCC stacking fault which separates partial dislocations) is introduced within the 
precipitate on the dislocation glide plane. As shown in Supplementary Table 1, HCP Co is energetically favored to FCC Co, and thus, additional work is required to transform the now HCP structure on the dislocation glide plane within the precipitate back to an FCC structure. This imparts resistance that acts against the trailing Shockley partial dislocation. The maximum force imparted on the trailing partial dislocation can be calculated as,

$$
F_{\max }=\frac{\pi R^{2}\left|\gamma_{\mathrm{SFE}}\right|}{b}
$$

where $R$ is the precipitate radius, $b$ is the dislocation Burgers vector and $\gamma_{\mathrm{SFE}}$ is the stacking fault energy, which defines the difference in energy between planar FCC and HCP stacking. Since HCP cobalt is energetically preferred at low temperatures, $\gamma_{\mathrm{SFE}}$ of the FCC phase is negative and the absolute value is introduced into Eq. (1). The CRSS on the dislocation due to the structural transformation force given by Eq. (1) is obtained from a conventional dispersed barrier hardening relationship [18],

$$
\tau_{\mathrm{CRSS}}=\frac{F_{\max }}{b L}
$$

where $L$ is the inter-particle spacing, which we take equal to the periodic spacing along the dislocation line less the precipitate diameter. Following Ashby [23], the critical stress for Orowan looping of an edge dislocation about an impenetrable obstacle is 


$$
\tau_{\mathrm{CRSS}}=\frac{G b}{2 \pi} \frac{1}{L} \ln \left(\frac{x}{r_{o}}\right)
$$

where $G$ is the shear modulus, which for our simulation geometry is that of the shear modulus on the $\{111\}$ plane in the $<110>$ direction, namely $\left(3 C_{44}\left(C_{11^{-}} C_{12}\right) /\left(C_{11^{-}}\right.\right.$ $\left.C_{12}+4 C_{44}\right)$ ) [24], $x$ is the particle diameter and $r_{o}$ represents the dislocation core radius, which we assume equals $\sqrt{2} b$.

The Gibbs free energy difference between HCP and FCC cobalt decreases linearly with increasing temperature until becoming zero at about $700 \mathrm{~K}$ [25]. Thus, we assume the same linear temperature dependence for the (negative) stacking fault energy, from the known value of $-37 \mathrm{~mJ} / \mathrm{m}^{2}$ at $0 \mathrm{~K}$ (Supplementary Table 1 ) to $0 \mathrm{~mJ} / \mathrm{m}^{2}$ at $700 \mathrm{~K}$. Fig. 5a plots the predicted CRSS, normalized to the maximum Orowan strength (Eq. 2, using elastic constants at $1 \mathrm{~K}$ ), due to precipitate strengthening obtained using equations (1) and (2) for a $3 \mathrm{~nm}$ diameter Co precipitate based on the (new) structural phase transformation precipitate strengthening mechanism (shown in black) and compares it to the Orowan strength obtained from Eq. (3) (shown in blue). In both calculations, we assume a temperature dependence of the copper elastic constants, consistent with the published values (Ref. 26). The resulting strengthening effect of the precipitate on dislocation motion should be the minimum CRSS between that given by Eqs. (1) and (2), which shows a strong temperature dependence and considerable softening for temperatures above about $250 \mathrm{~K}$. The predicted transformation between Orowan looping and a shear bypass mechanisms occurs at a temperature of about $220 \mathrm{~K}$, which is very consistent with the bypass mechanism shift observed for an edge dislocation with a $3 \mathrm{~nm}$ coherent Co 
precipitate in the MD simulations, as shown in Fig. 5b. At low temperatures, the structural phase transformation precipitate strengthening from Eq. (2) is greater than that associated with the Orowan strength, and therefore a dislocation bypass mechanism by Orowan looping would be expected as the path of least resistance, consistent with the MD simulation results. The strong linear temperature resistance of the structural phase transformation precipitate strengthening mechanism produces a cross over in the minimum CRSS between the two mechanisms curves at about $220 \mathrm{~K}$. Indeed, for temperatures of 300 and $400 \mathrm{~K}$, the MD simulations reveal that the dislocation interacts and bypasses the precipitate with the expected shear mechanism (Fig. 5b).

Furthermore, we can incorporate an estimate of the phonon drag, or inertial effect, on the dislocation motion by assigning an effective drag stress on the dislocation according to the well-known relation [22],

$$
\tau_{\text {drag }}=\frac{B v}{b}
$$

where $B$ is the Friction coefficient, with units of $\mathrm{Ns} / \mathrm{m}^{2}, v$ is the average dislocation velocity and $b$ is the Burgers vector. We have used MD simulations to evaluate the temperature dependence of $\mathrm{B}$, which ranges from about $2 \times 10^{-5} \mathrm{Ns} / \mathrm{m}^{2}$ at $10 \mathrm{~K}$ to $3 \times 10^{-5}$ $\mathrm{Ns} / \mathrm{m}^{2}$ at $500 \mathrm{~K}$ as shown in Supplementary Fig. 3, and used an average dislocation velocity of $750 \mathrm{~m} / \mathrm{s}$, based on observations from MD simulations, to estimate a drag stress contribution to the overall CRSS. In Fig. 6, this effective resistance to dislocation motion due to phonon drag is plotted by the blue squares, and is motivated by the analysis of Nembach and co-workers [10,11]. The black circles in Fig. 6 represent the 
normalized temperature dependent precipitate strengthening obtained from the minimum resistance of the two strengthening mechanisms from Fig. 5a. A root sum square superposition of these two contributions to the dislocation resistance results in the curve plotted with red diamonds as the overall CRSS for a $\mathrm{Cu}-\mathrm{Co}$ alloy containing $3 \mathrm{~nm}$ diameter, coherent cobalt precipitates and an obstacle spacing of $26.5 \mathrm{~nm}$. The qualitative features of this predicted CRSS are in remarkably good agreement with the experimentally determined temperature dependence of the CRSS of $\mathrm{Cu}-\mathrm{Co}$ alloys reproduced in Fig. 1. Indeed, we believe that a combination of a dynamic effects on the dislocation motion due to phonon drag and a temperature dependent precipitate strengthening can fully explain the observed temperature dependence of the $\mathrm{Cu}-\mathrm{Co}$ system, in which a peak CRSS is observed at temperatures between 200 and $250 \mathrm{~K}$, and that the CRSS at $500 \mathrm{~K}$ is approximately the same order of magnitude as that at $10 \mathrm{~K}$.

The temperature dependence of the normalized CRSS predicted in Fig. 6 is in excellent agreement with both the MD simulations and the experimental results $[10,11]$. Thus the MD simulations reveal the existence of a new precipitate strengthening mechanism in the $\mathrm{Cu}$-Co alloy system, which produces a strongly temperature dependent precipitate strengthening, in which a reversible structural phase transformation results in an Orowan looping bypass mechanism of the trailing partial dislocation at low temperature. When combined with the dynamic dislocation effects described by Nembach and co-authors [10,11], using a simplified model of a phonon drag contribution to the CRSS, Fig. 6 shows that the predicted temperature dependence of the CRSS measured in $\mathrm{Cu}-\mathrm{Co}$ alloys with small coherent precipitates agrees well with experimental measurements and MD simulations. Furthermore, the relative absence of temperature 
dependence in alloys with larger incoherent precipitates, can also be understood, since incoherent precipitates with dislocation bypass occurring by an Orowan looping mechanism would have a much weaker temperature dependence than the mixed-mode bypass observed for the smaller, coherent precipitates.

\section{Conclusions}

Molecular dynamics simulations have been performed to investigate the dislocation interaction and bypass mechanism from coherent, spherical cobalt precipitates in copper. The results of these simulations can be understood based on the underlying dislocation interaction mechanisms with precipitates, and a model has been developed to capture the temperature dependence of the critical resolved shear stress of $\mathrm{Cu}-\mathrm{Co}$ alloys through the superposition of precipitate strengthening and phonon drag. The major findings are:

- MD simulations reveal a strongly temperature dependent precipitate strength for a coherent, $3 \mathrm{~nm}$ diameter Co precipitate in $\mathrm{Cu}$, and the interaction strength also differs for the leading and trailing partial dislocations of both screw and edge dislocations.

- Below $300 \mathrm{~K}$, both the trailing partial dislocations of screw and edge dislocations bypass the coherent, $3 \mathrm{~nm}$ diameter cobalt precipitate by Orowan looping. At temperatures above $300 \mathrm{~K}$, the interaction is governed by the expected shear mechanism.

- The Orowan looping results from the structural transformation of the precipitate into the more stable hexagonal close packed structure on the glide plane, and is reversible.

- A model for the CRSS of $\mathrm{Cu}-\mathrm{Co}$ alloys has been developed based on the superposition of a temperature dependent precipitate strengthening and a contribution 
from phonon drag. The model agrees well with both experimental data and these MD simulations.

\section{Acknowledgements}

The authors acknowledge many helpful discussions with Professors Ian Robertson (University of Illinois at Urbana-Champaign) and David Rodney (Génie Physique et Mécanique des Matériaux) and financial support from the NSF under contracts NSF DMR 0244562, NSF DMR 0548259, and the U.S. Department of Energy, Office of Fusion Energy Sciences under grant DOE-DE-SC0006661. J.H.S. acknowledges the support from the Convergence Agenda Program (CAP) of the National Research Council of Science and Technology of Korea.

\section{References}

[1] S.S.P. Parkin, R. Bhadra, K.P. Roche, Oscillatory magnetic exchange coupling though thin copper layers, Phys. Rev. Lett. 66 (1991) 2152-2155.

[2] S. Serrano-Guisan, G. di Domenicantonio, M. Abid, J.-P. Abid, M. Hillenkamp, L. Gravier, J.-P. Ansermet, C. Félix, Enhanced magnetic field sensitivity of spindependent transport in cluster-assembled metallic nanostructures, Nature Mater. 5 (2006) 730-734.

[3] J.D. Livingston, J.J. Becker, A study of precipitation-hardening employing magnetic measurements, Trans. Metall. Soc. AIME 212 (1958) 316-320.

[4] V.A. Phillips, Electron microscope observations on moiré fringes and interfacial dislocations at cobalt precipitates in copper, Acta Metall. 14 (1966) 271-278. 
[5] F.J. Humphreys, The interaction of dislocations with coherent cobalt particles in a copper matrix, Acta Metall. 16 (1968) 1069-1073.

[6] A.J. Ardell, The effect of volume fraction on particle coarsening: theoretical considerations, Acta Metall. 20 (1972) 61-71.

[7] S. Satoh, W.C. Johnson, Comparison between calculated and observed elastically induced precipitate shape transitions ion a $\mathrm{Cu}-2$ at. pet Co alloy, Metall. Trans. A 23A (1992) 2761-2773.

[8] M. Takeda, N. Suzuki, G. Shinohara, T. Endo, J. van Landuyt, TEM study on precipitation behavior in Cu-Co alloys, Phys. Stat. Sol. A 168 (1998) 27-35.

[9] V. Mohles, Computer simulations of the glide of dissociated dislocations in lattice mismatch strengthened materials, Mater. Sci. Eng. A 324 (2002) 190-195.

[10] N. Büttner, E. Nembach, Low temperature anomalies of precipitation hardening in the system copper-cobalt, Z. Metallkd. 76 (1985) 82-84.

[11] K.-D. Fusenig, E. Nembach, Dynamic dislocation effects in precipitation hardened materials, Acta Metall. Mater. 41 (1993) 3181-3189.

[12] H.J. Frost, M.F. Ashby, Deformation-Mechanism Maps: The Plasticity and Creep of Metals and Ceramics, Ch. 2, Pergamon Press, Oxford, 1982.

[13] T. Diaz de la Rubia, M.W. Guinan, Progress in the development of a molecular dynamics code for high-energy cascade studies, J. Nucl. Mater. 174 (1990) 151-157.

[14] M.S. Daw, M.I. Baskes, Embedded-atom method: Derivation and application to impurities, surfaces, and other defects in metals, Phys. Rev. B 29 (1984) 6443-6453.

[15] S.M. Foiles, M.I. Baskes, M.S. Daw, Embedded-atom-method functions for the fcc metals $\mathrm{Cu}, \mathrm{Ag}, \mathrm{Au}, \mathrm{Ni}, \mathrm{Pd}, \mathrm{Pt}$, and their alloys, Phys. Rev. B 33 (1986) 7983-7991. 
[16] R. Pasianot, E.J. Savino, Embedded-atom-method interatomic potentials for hcp metals, Phys. Rev. B 45 (1992) 12704-12710.

[17] K. Nordlund, R.S. Averback, Inverse Kirkendall mixing in collision cascades, Phys. Rev. B 59 (1999) 20-23.

[18] E. Nembach, Particle Strengthening of Metals and Alloys, John Wiley \& Sons, New York, 1997.

[19] A.S. Clarke, H. Jónsson, Structural changes accompanying densification of random hard-sphere packings, Phys. Rev. E 47 (1993) 3975-3984.

[20] D. Faken, H. Jónsson, Systematic analysis of local atomic structure combined with 3D computer graphics, Comp. Mater. Sci. 2 (1994) 279-286.

[21] J. Li, AtomEye: an efficient atomistic configuration viewer, Model. Simul. Mater. Sci. Eng. 11 (2003) 173-177.

[22] J.P. Hirth, J. Lothe, Theory of Dislocations, 2nd ed., Krieger Publishing Company, Malabar, 1992.

[23] M.F. Ashby, Results and consequences of a recalculation of the Frank-Read and the Orowan stress, Acta Metall. 14 (1966) 679-681.

[24] C.N. Reid, Deformation Geometry for Material Scientists, Pergamon Press, Oxford, 1973.

[25] A.T. Dinsdale, SGTE data for pure elements, CALPHAD 15 (1991) 317-425.

[26] G. Simmons, H. Wang, Single Crystal Elastic Constants and Calculated Aggregate Properties: A Handbook, 2nd ed., MIT Press, Cambridge, 1971. 


\section{Figure legends}

Fig. 1. Temperature dependence of CRSS for $\mathrm{Cu}$-Co alloys. Triangles, diamonds, circles and squares represent $2.23,1.28,0.93$ and $0 \%$ Co contents, respectively.

Fig. 2. Snapshots of the MD simulation of the edge dislocation glide at $300 \mathrm{~K}$ at (a) 16.0 , (b) 22.8, (c) 40.0, (d) 44.0, (e) 44.6 and (f) 50.0 ps after applying a shear stress of $100 \mathrm{MPa}$. Yellow and gray spheres represent $\mathrm{Cu}$ and $\mathrm{Co}$ atoms, respectively.

Fig. 3. Snapshots of the MD simulations of the edge dislocation glide at $100 \mathrm{~K}$ at (a) 15.0, (b) 22.2, (c) 38.8, (d) 41.2 (e) 41.4 and (f) 45.2 ps after applying a shear stress of $100 \mathrm{MPa}$. Yellow and gray spheres represent $\mathrm{Cu}$ and $\mathrm{Co}$ atoms, respectively.

Fig. 4. Snapshots of the MD simulations of the screw dislocation glide at $100 \mathrm{~K}$ at (a) 25.6, (b) 45.2, (c) 47.8 and (d) 50.0 ps after applying a shear stress of $100 \mathrm{MPa}$. Yellow and gray spheres represent $\mathrm{Cu}$ and $\mathrm{Co}$ atoms, respectively.

Fig. 5. (a) Model predictions for the two bypass mechanisms, structural strengthening (black circles) versus the Orowan looping mechanism (blue squares) and (b) mechanism map showing the dislocation bypass mechanism versus temperature and applied stress, as observed by MD simulations of the interaction of a $3 \mathrm{~nm}$, coherent Co precipitate with an edge dislocation. Also shown is the effective CRSS obtained from the MD simulations.

Fig. 6. Predicted temperature dependent normalized CRSS for a $\mathrm{Cu}-\mathrm{Co}$ alloy containing 3 nm diameter, coherent Co precipitates. 


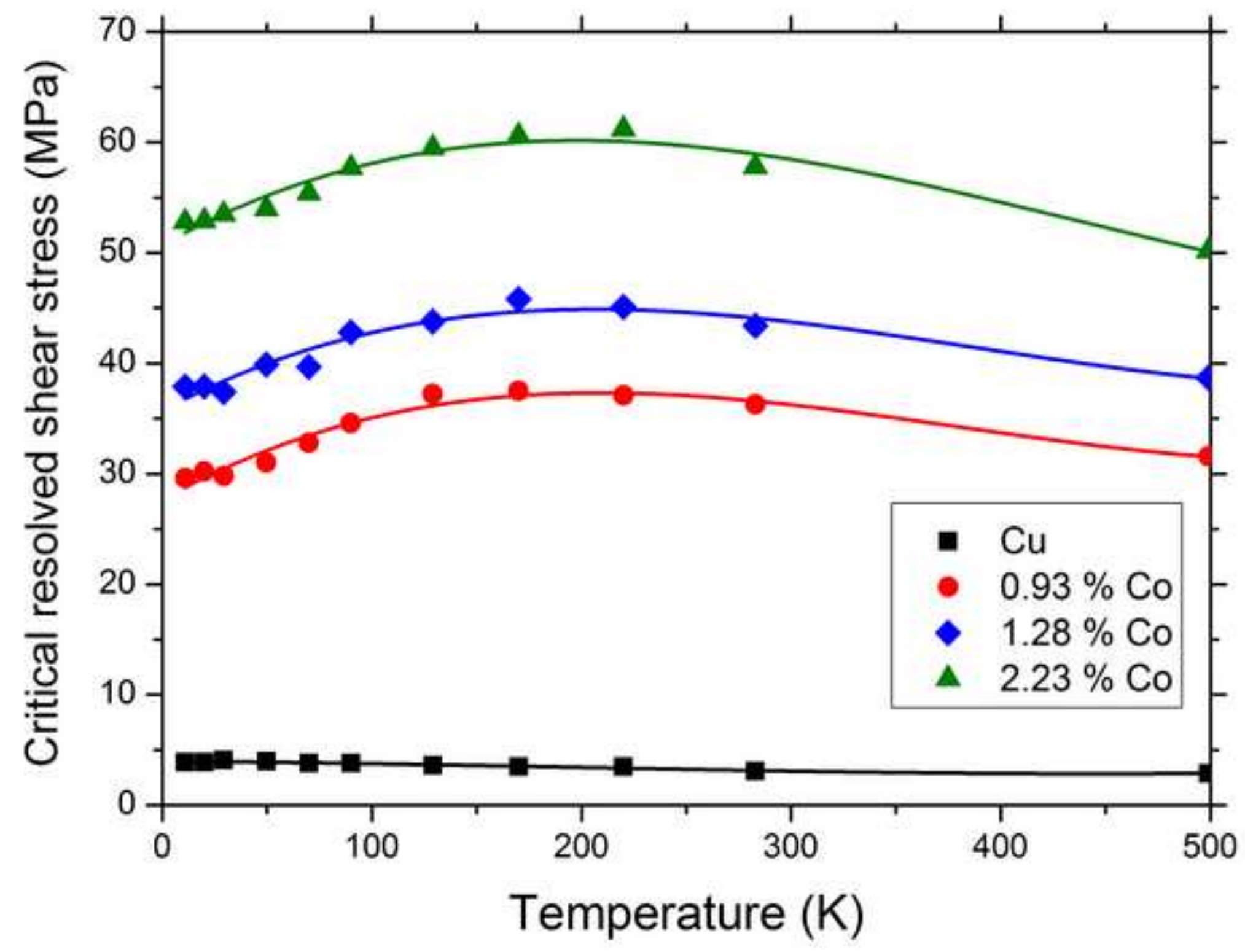



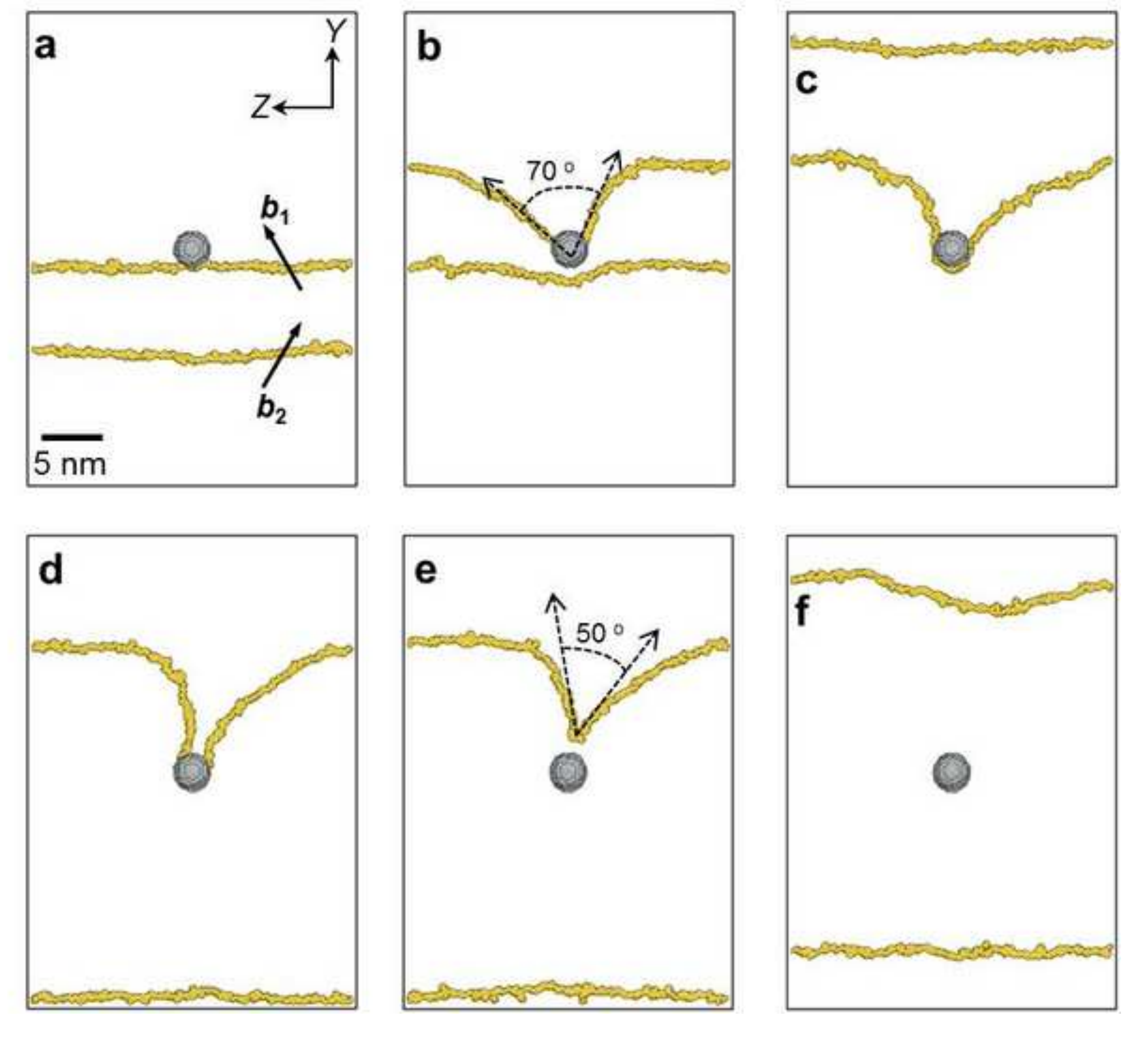

Fig. 2

.
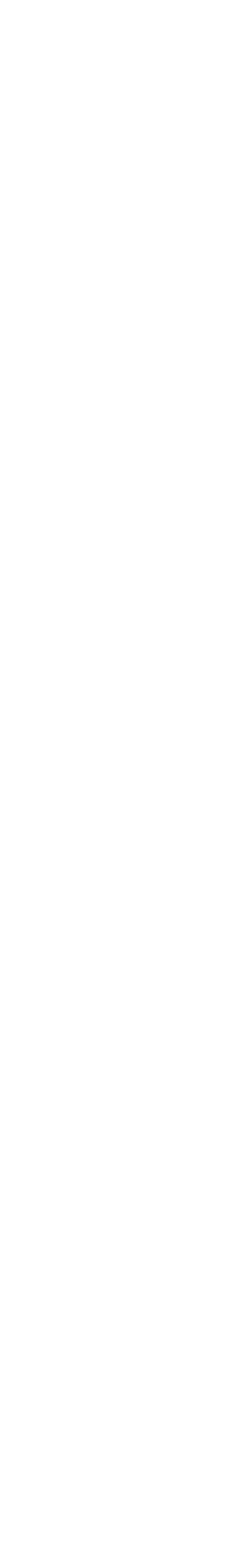

.



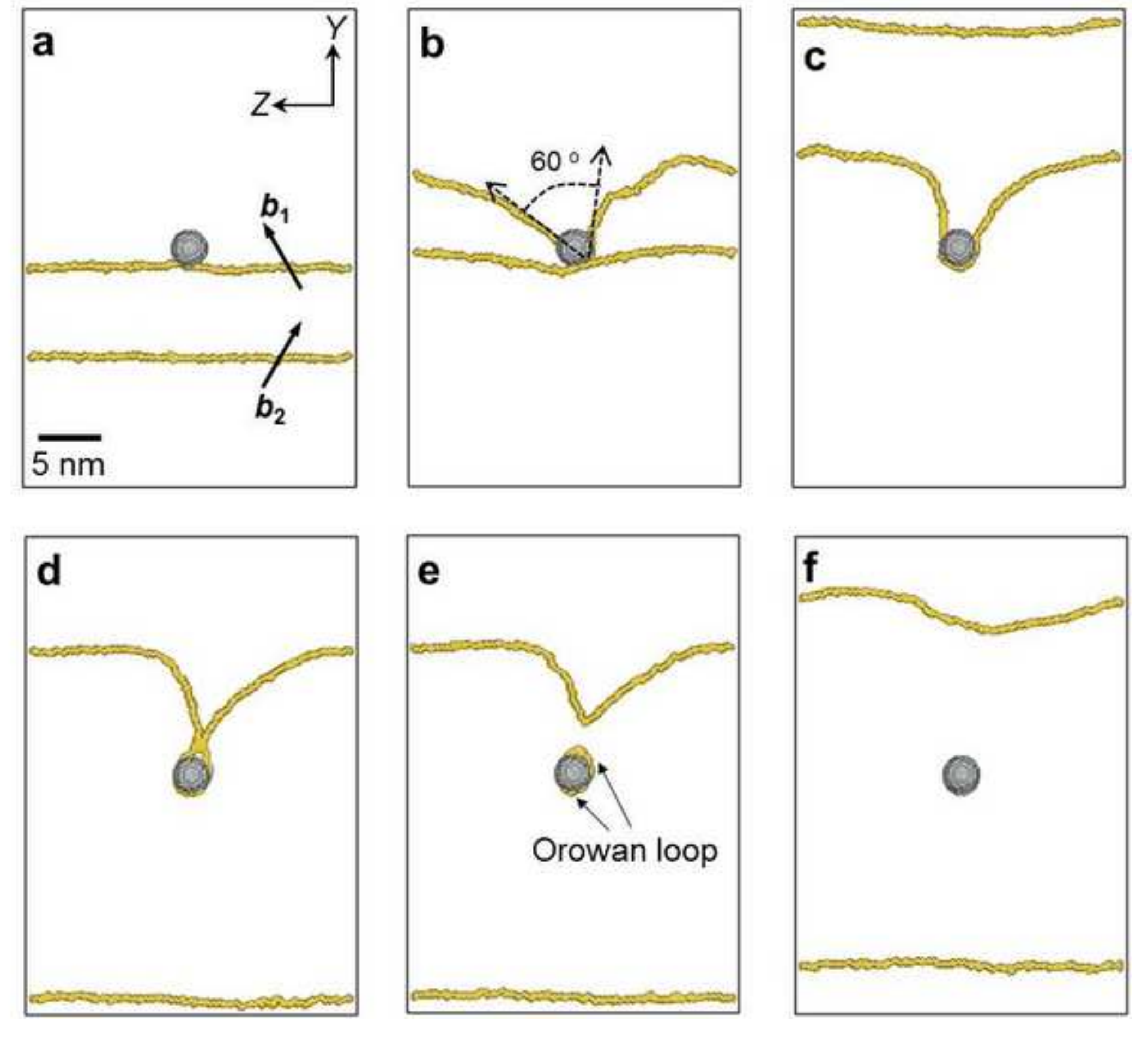

,



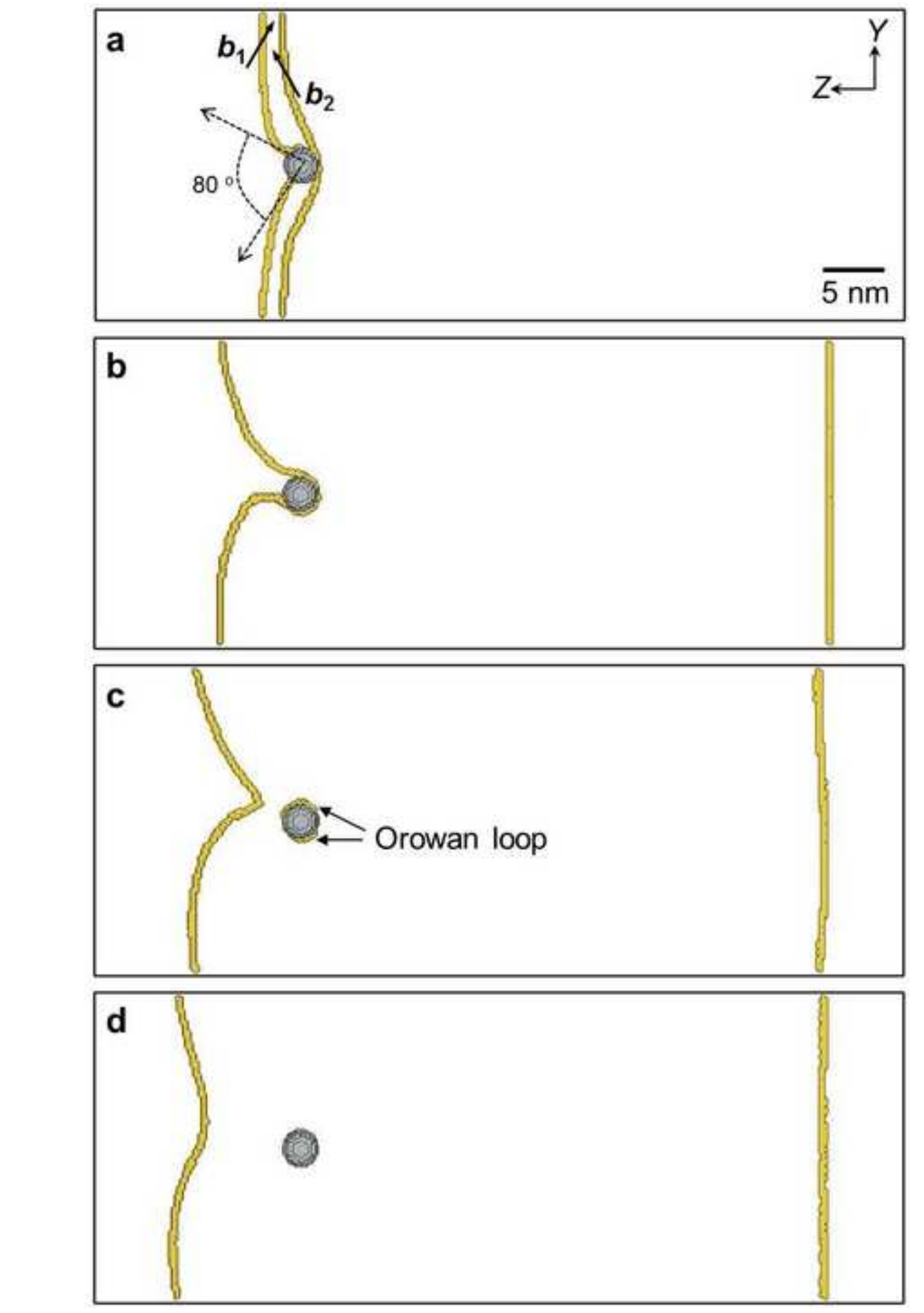

Fig. 4

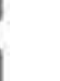

Fig. 4

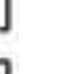




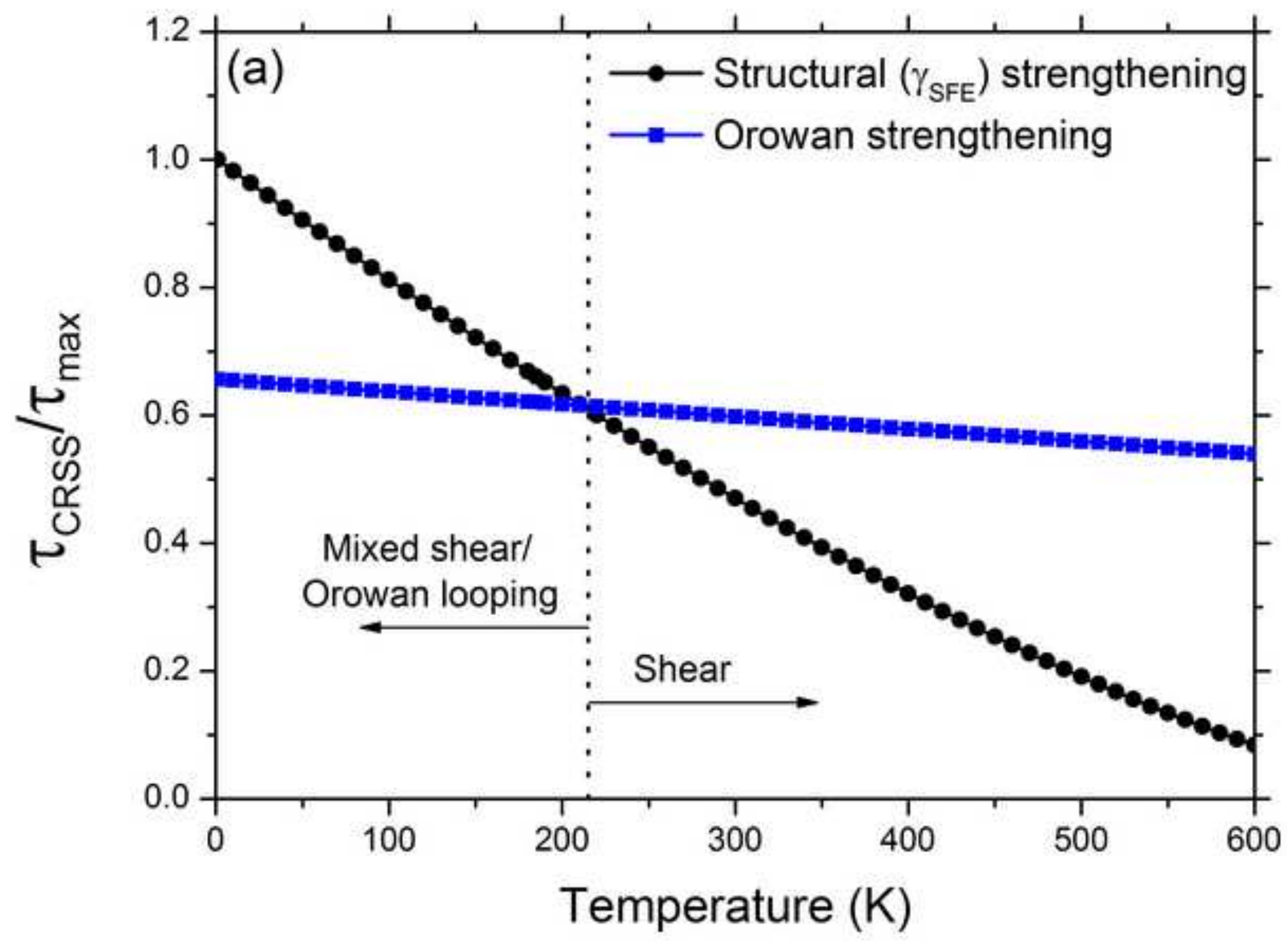




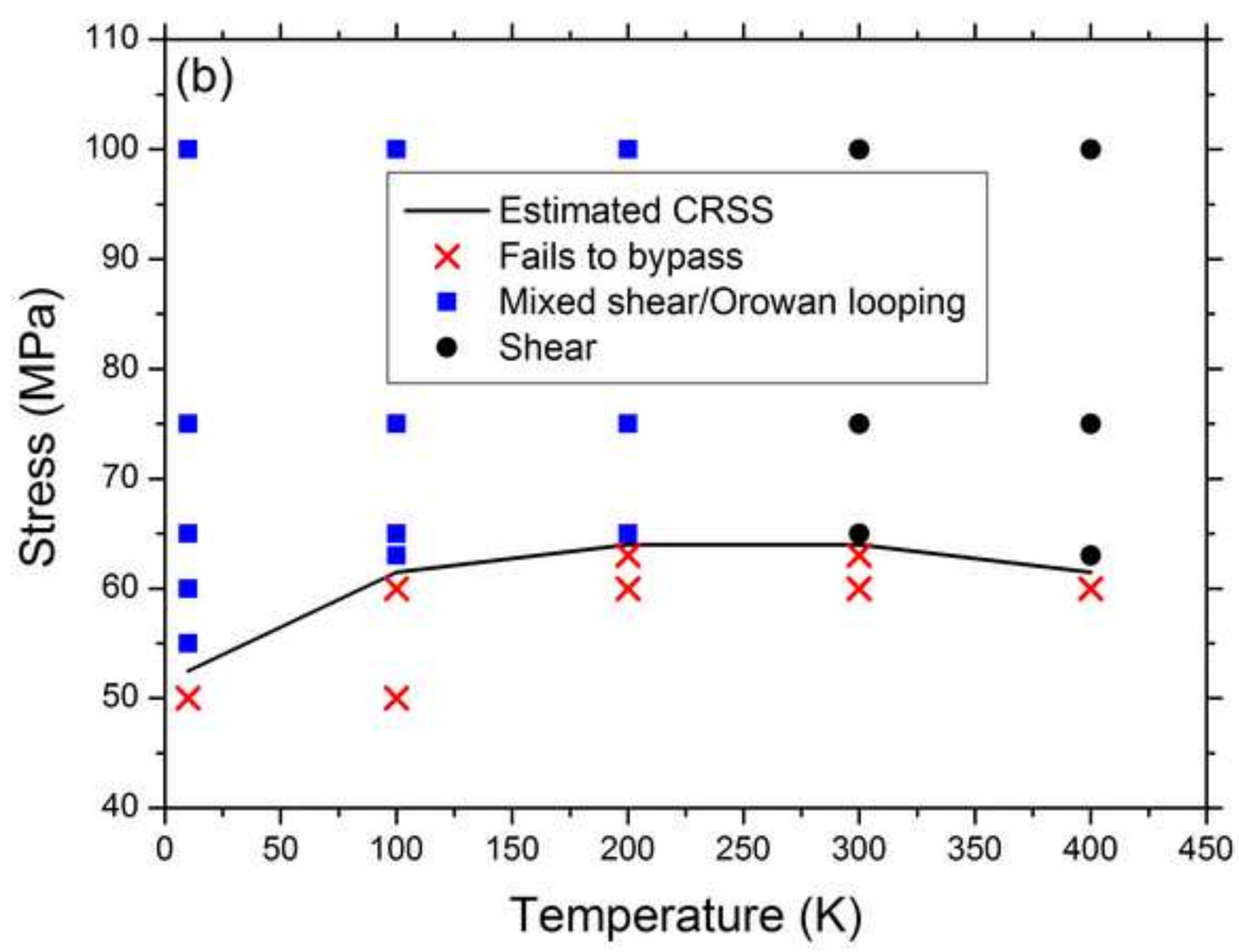




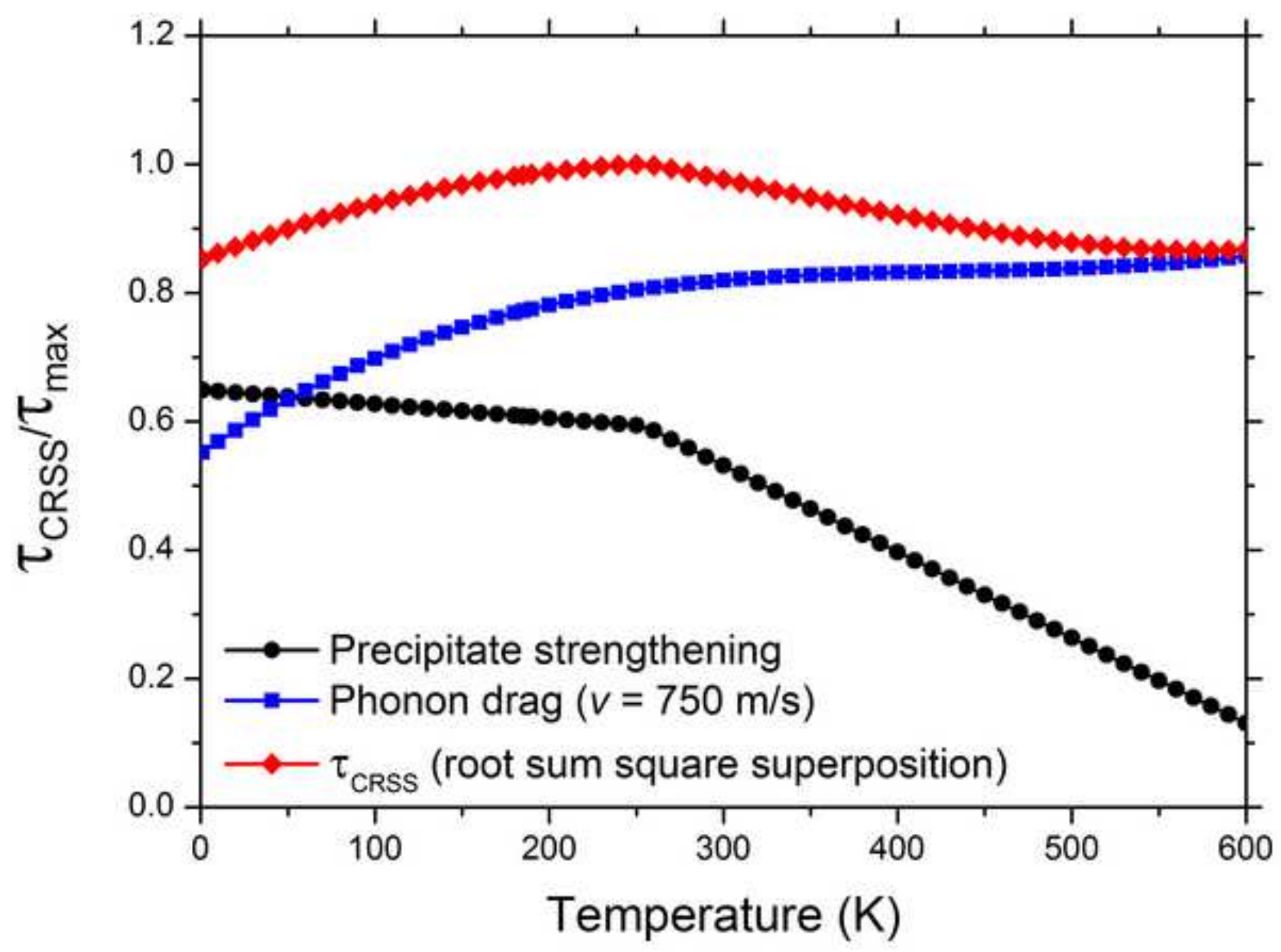




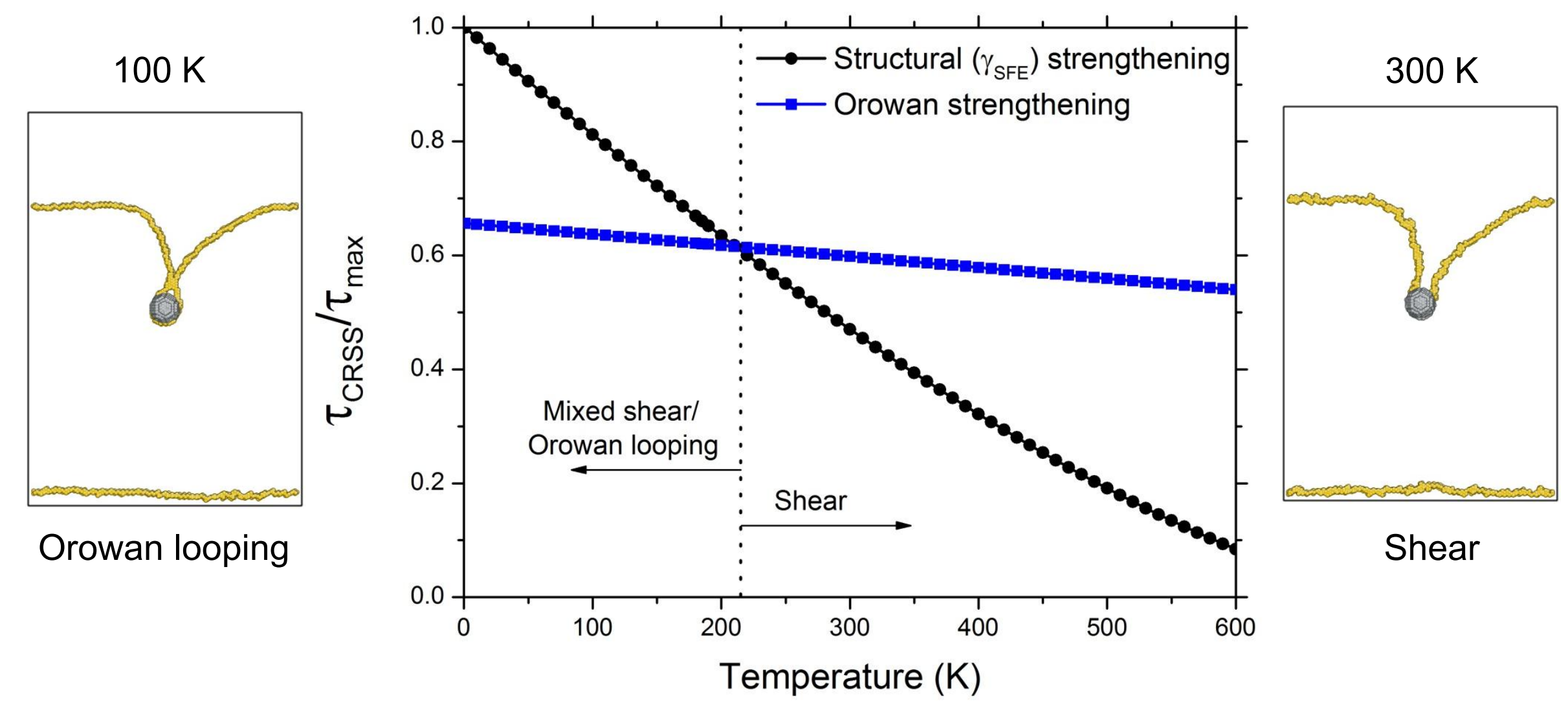

